\title{
Paese che vai usanza che trovi, tra cosmo e campanile
}

"So many countries, so many customs". Between the world and the hometown

\section{Pietro Clemente}

\section{(2) OpenEdition \\ 12 Journals}

Edizione digitale

URL: http://journals.openedition.org/aam/809

DOI: $10.4000 /$ aam.809

ISSN: 2038-3215

Editore

Dipartimento Culture e Società - Università di Palermo

Notizia bibliografica digitale

Pietro Clemente, « Paese che vai usanza che trovi, tra cosmo e campanile », Archivio antropologico mediterraneo [Online], Anno XXI, n. 20 (2) | 2018, online dal 31 décembre 2018, consultato il 23 septembre 2019. URL : http://journals.openedition.org/aam/809; DOI : 10.4000/aam.809

Questo documento è stato generato automaticamente il 23 septembre 2019.

Archivio antropologico mediterraneo 


\title{
Paese che vai usanza che trovi, tra cosmo e campanile
}

"So many countries, so many customs". Between the world and the hometown

\author{
Pietro Clemente
}

\section{NOTA DELL'EDITORE}

Lectio magistralis tenuta in occasione del conferimento del Premio Cocchiara 2018 a

Mistretta (Messina), il primo dicembre 2018. Ringraziamo l'Autore per averne consentito la pubblicazione.

\section{Un villaggio vivente}

1 Al cuore del messaggio antropologico c'è il tema della diversità culturale come nel proverbio: "Paese che vai, usanza che trovi". C'è anche il modo di dire "Il mondo è bello perché è vario" a suggerire il valore della pluralità culturale. Oggi la modernizzazione tende e uguagliare tutto e tutti, e tante volte ci sembra di non essere più individui particolari ma di finire per diventare degli individui-massa.

2 Per i giovani dare valore alle differenze culturali a partire dal proprio paese, dai giochi tradizionali alla lingua locale, ai canti è cosa assai importante. I giovani a mio avviso non debbono perdere la memoria locale per cercare solo la vita delle città e la modernità, perché rischiano così di perdere tutto. Come ha scritto l'antropologo Ernesto De Martino:

alla base della vita culturale del nostro tempo sta l'esigenza di ricordare una "patria" e di mediare - attraverso la concretezza di questa esperienza - il proprio rapporto col "mondo". Coloro che non hanno radici, che sono cosmopoliti, si avviano alla morte della passione e dell'umano: per non essere provinciali, occorre possedere un villaggio vivente della memoria a cui l'immagine e il cuore tornano sempre di nuovo, e che l'opera di scienza o di poesia riplasma, in voce universale [...] (De Martino 1959: 151). 


\section{Piccoli paesi}

Mi occupo in questi anni di una 'rete dei piccoli paesi' che rischiano di scomparire perdendo tutti gli abitanti e la memoria del territorio. Spesso sono i giovani che non vogliono restare perché pensano che nelle loro terre non ci sia nulla. Perché finiscono per avere una mentalità cittadina e non capire il valore della diversità. Certo, nei paesi è spesso evidente che non ci sono occasioni di lavoro, centri di esperienza, snodi di relazioni che facilitino la creazione di un curriculum di una prospettiva professionale. Ed è giusto che dai paesi si parta, ma senza miti e sapendo che lo sguardo sul futuro spinge a non abbandonare la strada del ritorno. L'Italia delle zone interne è la vera risorsa del nostro paese e chi ci vive fa un grande sforzo per difendere boschi, fiumi, prodotti biologici, forme di vita solidali, storie e forme di sapere sia materiale che simbolico che esiste solo nei luoghi e luogo per luogo.

Oggi che la cosa più importante è avere una 'coscienza di luogo' (Magnaghi 2010), Mistretta è un luogo importante per riflettere su questa coscienza di luogo. Perché è un antico centro, quasi una capitale per i Nebrodi e per quel Valdemone che Giuseppe Cocchiara aveva studiato con grande cura; ma nel tempo il Comune è passato da circa 15.000 abitanti ai 5.000 di ora. Ha perso il palazzo di giustizia e rischia di perdere l'ospedale; così anche questo straordinario luogo di montagna rischia di soffrire per la perdita dei servizi, come i più piccoli paesi.

Penso che abbia ragione il presidente Mattarella che questa estate ha detto in una intervista fatta in Sardegna:

Non mi stanco mai di ripetere che i cittadini italiani hanno gli stessi diritti, indipendentemente dalla loro collocazione geografica. E di sollecitare l'attenzione e il sostegno degli organi centrali di governo nei confronti delle isole minori, dei territori montani e delle zone interne del nostro Paese: $\mathrm{i}$ loro abitanti - tenendo vivi e ben curati i loro luoghi - svolgono un ruolo fondamentale a beneficio di tutta la comunità nazionale. [...] È indispensabile - per ragioni di eguaglianza e per interesse nazionale - assicurare agli abitanti di quelle località servizi non dissimili da quelli di cui possono avvalersi i loro concittadini delle grandi città ${ }^{\text {. }}$

Credo che i giovani debbano essere i primi a battersi per la parità di diritti. $\mathrm{E}$ a riconoscere il valore della propria storia, insieme a quello del valore delle nuove tecnologie che consentono di essere altrove anche senza muoversi e che hanno reso più semplice il viaggio e più vicino il mondo.

In questo senso è importante 'mettere il centro in periferia', cioè trovare una nuova dimensione dell'Italia in cui si guardi al domani non più dalle grandi città, ma dai piccoli paesi e dalle zone interne, perché questo nuovo sguardo serve non solo a restituire ad essi una democrazia piena, ma anche alle città delle fonti di qualità della vita e della natura cui ricorrere, verso le quali tornare ${ }^{2}$.

\section{Amastra}

7 Qui nel territorio dei Nebrodi e a Mistretta ci sono stati tanti studi, da quelli di Giuseppe Cocchiara a quelli, ad esempio, di Antonino Buttitta e di Salvatore D'Onofrio, inoltre c'è un museo ben realizzato e importante che è stato allestito con la regia di Sergio Todesco: il Museo Regionale delle tradizioni silvo-pastorali, e c'è il lavoro di 
tanti anni del gruppo di folklore Amastra. Ci sono quindi le condizioni per riconoscere il valore della cultura locale e trasmetterlo alle nuove generazioni perché lo vivano a modo loro, creativamente, in modo adeguato ai loro bisogni materiali e simbolici. Questo patrimonio che viene lasciato ai giovani non è più solo 'il passato' ma è anche il presente e il futuro, perché può servire a inventarsi una vita nuova qui in montagna. Nei paesi dell'America e dell'Oceania dove il mondo occidentale ha conquistato e sottomesso popoli locali ricchi di culture diverse, è successo che le culture locali anziché scomparire sotto il peso della modernità, sono rinate in forma nuova e si sono adattate alla vita di oggi. Sono queste forme di resistenza delle culture locali che impediscono alla modernità di uguagliare tutto e di rendere anonimo il mondo degli uomini. Uno studioso americano, James Clifford, ha usato l'espressione "Diventare indigeni nel XXI secolo" per indicare la capacità di vivere oggi nel nostro mondo portando con sé le differenze culturali come parte della vita attuale (Clifford 2013). Ci sono tanti elementi a Mistretta perché, sotto lo sguardo di Giuseppe Cocchiara, questa comunità si faccia 'indigena del nuovo millennio'. Dice ancora Clifford che oggi “il passato si trova dove si cerca il futuro". È quasi un enigma che voglio lasciarvi come compagnia e guida per un uso creativo e attuale della cultura popolare e della memoria del territorio.

\section{Dietro le quinte}

8 In queste cose che propongo non ci sono solo i miei pensieri, ma ci sono gli studi che ho fatto, i miei maestri che mi hanno indirizzato, e quindi desidero presentare ciò che c'è dietro le quinte di un ragionamento semplice sulla diversità culturale oggi. Il Premio Cocchiara è in un certo senso un 'premio alla carriera', e cercherò di presentarvi le principali tradizioni di pensiero ed esperienze teoriche ed etnografiche che hanno segnato il mio percorso, che hanno fatto la mia carriera. È stato il mio Maestro di studi Alberto Mario Cirese a suggerire che le nostre discipline demo-etno-antropologiche si collocano tra i due proverbi: 'Paese che vai usanza che trovi', e l'altro opposto e complementare 'Tutto il mondo è paese'. Egli aveva una predilezione per le invarianze $\mathrm{e}$ per il secondo dei due proverbi, io invece per la diversità e per il primo proverbio. Ma egli, che sentiva l'eredità e il valore dell'essere 'provinciali', ha anche riflettuto molto sui temi che sintetizzò in queste brevi frasi: 'con la testa nel mondo e il cuore nel borgo', 'con la testa nel mondo e i piedi nel borgo', e una sua riflessione sulle patrie culturali conteneva l'idea della vita culturale che si dispiega 'tra cosmo e campanile' (Cirese 2003). Voleva intendere che la vita degli uomini si dispiega nella sostanziale unità del genere umano, e insieme nella pluralità dei modi di vivere. Ma voleva suggerire anche di non accentuare mai una sola prospettiva: mettere al centro il locale, senza collocarlo nel quadro generale e viceversa. Diceva anche che gli studi sul luogo non si fanno con le teorie del luogo, come a dire che la conoscenza è sempre legata al mondo degli studi e non è mai localista. $\mathrm{E}$ che la centralità che hanno per noi oggi il luogo, la località, la 'perifericità' si basa su pensieri e teorie che vengono dal dibattito sulle prospettive della nostra civiltà. Sono i temi che anche io ho cercato di trattare in tanti scritti e in particolare nella mia recente esperienza della 'rete dei piccoli paesi'. Sia Cirese che De Martino hanno insegnato a Cagliari, dove io mi sono laureato. Anche se il dialogo tra Alberto Cirese e Ernesto De Martino era ormai interrotto quando nell'Università di Cagliari si formò una nuova generazione di antropologi, molti di noi, allievi di Cirese, mantennero i legami anche col pensiero di De Martino ${ }^{3}$; in questo 
ambito di studi ed etnografie locali io ho finito per considerare quasi un programma etico e di politica della ricerca questo piccolo racconto di fondazione dello studioso napoletano:

Io entravo nelle case dei contadini pugliesi come un "compagno", come un cercatore di uomini e di umane dimenticate istorie, che al tempo stesso spia e controlla la sua propria umanità, e che vuol rendersi partecipe, insieme agli uomini incontrati, della fondazione di un mondo migliore, in cui migliori saremmo diventati tutti, io che cercavo e loro che ritrovavo (De Martino 1953: 316).

\section{Territorialisti}

Il tema della 'coscienza di luogo' è relativamente nuovo, forse tutti gli antropologi che fanno etnografie locali maturano questo tipo di coscienza, ma in realtà, alle origini di questa prospettiva che può essere definita 'territorialista' c'è la crisi del marxismo e del suo modello progressista e economicista. Per me questa consapevolezza ha cominciato a maturare dopo il 1984 grazie anche alla riflessione di Gianluigi Bravo e di Piercarlo e Renato Grimaldi in dialogo con Luciano Gallino che elaborarono un modello duttile di analisi delle formazioni economico sociali che confluì nel volume di Gian Luigi Bravo Festa contadina e società complessa (Milano, Angeli, 1984). Nel contempo mi portavano fuori del modello marxista anche gli studi sociologici di Arnaldo Bagnasco e di altri sociologi della 'terza Italia', ma anche classici del pensiero sociologico come il lavoro di Pierre Bourdieu sulla pratica ${ }^{4}$, dai quali ebbi anche il primo incontro con Wittgenstein usato come strumento critico dei concetti forti in antropologia. Ma è alla fine degli anni Ottanta che potei avere il senso di un 'orientamento' in questo ambito avendo l'occasione di un incontro e una collaborazione con uno degli economisti che teorizzarono i distretti industriali lavorando anche sulle risorse culturali e simboliche degli attori sociali. Si tratta di Giacomo Becattini, dal cui dialogo con l'urbanista Alberto Magnaghi nascono le prospettive teoriche della Società dei Territorialisti, riunitasi a congresso a Firenze nel 2011 (cfr. Magnaghi 2012). Un volumetto dialogante, tra Magnaghi e Becattini, uscito poco prima della morte dell'economista, resta un riferimento importante per un approccio alle culture locali che recupera gli studi delle tradizioni popolari e dell'antropologia dell'Italia, ma li ri-orienta nell'ambito di un approccio territorialista (Becattini 2015). Sono poi gli studi di Magnaghi a sottolineare anche un passaggio teorico epocale, quello dalla 'coscienza di classe' alla 'coscienza di luogo', che comporta una diversa strategia e immaginazione di futuro per gli attori sociali. Ecco un passo di Magnaghi:

Il ritorno al territorio non significa il ritorno a forme di vita particolari o a sistemi socioeconomici e culturali del passato ma rinvia in generale al ritorno a condizioni di vita sostenibili e durevoli della specie umana sulla terra. Rinvia a delle condizioni dell'ambiente umano che sono in via di degrado crescente, a causa dei vasti processi di deterritorializzazione, di esodo e di urbanizzazione del mondo, ad opera di agenti del capitale tecno-finanziario mondiale (Magnaghi 2017: 8; traduzione mia).

In effetti, quando propongo ai giovani di assumere l'esperienza locale come un riferimento della propria storia, delle partenze e dei ritorni, e le tradizioni e i saperi locali come una risorsa, il riferimento è al pensare strategicamente il futuro a partire dai territori abbandonati, visti come grande risorsa entro un processo possibile di inversione del rapporto con la città e del riabitare l'Italia oggi marginale, vista come 
luogo forte di risorse naturali, di prodotti e gastronomie di qualità, di diversità e creatività culturali ${ }^{5}$.

\section{Indigeni del XXI secolo}

11 In questa prospettiva critica si colloca per me il valore del ritrovare Antonio Gramsci, non più solo nelle pagine sul folklore, discusse per tanti anni dagli studiosi italiani di tradizioni popolari, ma soprattutto in quelle assai più numerose e varie sugli strati, le culture, gli intellettuali, la società civile, le alleanze:

In Oriente lo Stato era tutto, la società civile era primordiale e gelatinosa; nell'Occidente tra Stato e società civile c'era un giusto rapporto e nel tremolio dello Stato si scorgeva subito una robusta struttura della società civile. Lo Stato era solo una trincea avanzata, dietro cui stava una robusta catena di fortezze e di casematte; più o meno, da Stato a Stato, si capisce, ma questo appunto domandava un'accurata ricognizione di carattere nazionale (Quaderni dal carcere 7, 866).

Gramsci 'ritrovato' è stato un bell'argomento anche di riflessione con Alberto Mario Cirese e Giorgio Baratta con i quali abbiamo costruito due numeri di Lares assai vivaci e riflessivi ${ }^{6}$. La ricchezza e le articolazioni complesse dell'economico, politico, culturale di Gramsci, non riducibili agli schematismi di vari interpreti neo-marxisti, restituiscono a Gramsci e al suo marxismo 'speciale' un ruolo nelle analisi della complessità e in specie della società civile in tutte le sue complesse mutazioni. È la strada di Gramsci che porta, almeno me, a James Clifford, e al suo lavoro sui processi di costruzione di identità all'interno del mondo contemporaneo.

Negli anni Novanta in cui, insieme a un bel gruppo redazionale senese, realizzammo la rivista Ossimori, venimmo criticati da alcuni studiosi italiani per avere investito sugli studi americani di Clifford Geertz e di James Clifford, ed altri, anziché sulla tradizione italiana. Io pensavo allora, e ancora oggi, che non c'erano autori più vicini alla tradizione italiana di questi americani, non solo perché erano nutriti di filosofie europee, ma anche talora - come nel caso di Clifford -, per avere studiato tanti anni in Europa e avere scritto su tematiche della tradizione antropologia europea. James Clifford, più giovane di me per nascita, lo ho sentito da subito contiguo, per i suoi libri ricchi di riferimenti al surrealismo, all'arte francese, e infine per i riferimenti a Gramsci che tornano in modo sistematico nel suo lavoro Returns, dal quale il tema degli 'indigeni del XXI' secolo è tratto. James Clifford legge Gramsci attraverso la interpretazione data da Stuart Hall, sociologo inglese-giamaicano, dando un particolare rilievo al concetto di 'articolazione'. Un concetto che suggerisce di osservare la società come insieme non organico, ma come assemblaggio composito di tratti che coabitano ed entrano in relazioni speciali. Se una società locale ha perso la competenza linguistica ma non quella nautica, o ha perso la danza ma non il cibo, se ha riletto i suoi modelli culturali alla luce della globalizzazione, non è per questo 'perduta', prigioniera del capitalismo e delle sue ideologie individualiste, ma esprime invece resistenza e capacità di costruzione di processi originali, nuove alleanze tra aspetti culturali e con nuovi soggetti. Su questi temi Clifford ha già espresso nei primi anni 2000 delle posizioni molto interessanti in specie nelle interviste in Ai margini dell'antropologia (Clifford 2004), e tra i suoi testi precedenti I frutti puri impazziscono (Clifford 1993) è stato un riferimento importante anche per il dibattito italiano. Personalmente debbo varie riflessioni anche sui temi delle culture locali, delle comunità, dell'ibridazione, al saggio ivi contenuto "Identità a Mashpee", che racconta come un gruppo tribale di nativi americani 
intraprese una causa per il riconoscimento dei diritti che spettavano loro come 'tribù', non ottenendo di essere legalmente e storicamente considerata tribù per la legge americana, ma al tempo stesso riuscendo a costruire - nel processo - una nuova identità culturale moderna, diversa, e in un certo senso 'resistente'. Nel libro Returns Clifford mostra come certe previsioni relative allo sviluppo capitalistico capace di cancellare identità, culture e territori, riplasmando tutto a propria immagine, non hanno funzionato, e come elementi di resistenza culturale, locale, territoriale si siano via via definiti e consolidati, e in grado oggi di riconoscersi in un 'movimento' culturale in cui l'essere indigeni non sta nella scelta tra purezza e tradimento, ma in una nuova modalità e presenza di identità ibride e di soggetti culturali articolati.

Ecco un passaggio di Returns:

Il parziale intreccio delle società indigene e locali nelle strutture globali non è il risultato di un lavoro di cancellazione non ancora completato dal sistema mondo. Esso ha un dinamismo proprio. Così come è stato dimostrato dalle etnografie storicamente più avvertite nel Pacifico, i movimenti contemporanei relativi all'identità, al Kastom (espressione pidgin per customs), e alla sovranità continuano trasformando le lunghe storie di conflitti e di interazioni (Clifford 2013: 57; traduzione mia).

14 Questo lavoro concorda con l'esperienza degli studiosi indigeni nel riconoscere forme di sopravvivenza culturale, di resistenza e di innovazione nel cambiamento dei contesti di performance e di alleanze. Le tradizioni articolano selettivamente il ricordo e connettono passati e presenti. Molti studi degli studiosi 'nativi' affermano che il "passato" nelle epistemologie indigene è dove uno cerca il "futuro". I movimenti sempre più forti di sovranità tribale del 1980 e 1990 mostrano, infine, che l'egemonia corrente - che viene chiamata neocolonialismo, postmodernità, globalizzazione, americanizzazione, o neoliberismo - è fratta e significativamente aperta a esiti diversi.

Io ho cercato dunque di connettere la tradizione italiana di studi locali con le riflessioni di un autore che ha presente soprattutto il mondo dei nativi americani e oceaniani. Ho cercato di portare in Europa le esperienze di altri continenti. Anche i nostri 'nativi' dei paesi e delle zone interne della varie regioni italiane nel momento in cui attivano, traendole dalla memoria di altre generazioni, comunità che producono carnevali, ritorni al paese, gastronomie, culture della montagna o del fiume, in qualche modo $s i$ fanno indigeni del nostro tempo. Mostrano praticamente che la diversità culturale, la creatività locale, la polifonia della società civile, sono temi all'ordine del giorno. Queste culture 'indigene' non sono né distorte o corrotte dal sistema neoliberista, né nascoste sotto la cenere pronte a rinascere, né in attesa di liberatori che vengono dall'esterno, sono invece vive, ibride, contemporanee e incerte nel loro andamento. In esse si possono vedere nuovi investimenti per il futuro.

Mi è sembrato con questi temi di mostrare in azione una tradizione di studi italiani, e un dialogo con gli studi del mondo globale, che può avere qualche riconoscibile utilità perfino per le nuove generazioni. Così, nel ricordare e ringraziare i miei Maestri ho cercato di portarli oltre il confine del tempo che hanno vissuto, confine sul quale infine anche io mi trovo. 


\section{BIBLIOGRAFIA}

Becattini G., 2015 La coscienza dei luoghi. Il territorio come soggetto corale, Roma, Donzelli.

Bourdieu P., 1980 Le Sens pratique, Minuit, Paris.

Cirese A.M., 2003 Tra cosmo e campanile. Ragioni etiche e identità locali, Siena, Protagon.

Clifford J., 1993 [ed. or. 1988] I frutti puri impazziscono. Etnografia, letteratura e arte nel secolo XX, Torino, Bollati Boringhieri.

- 2004 [ed. or. 2003] Ai margini dell'antropologia, Roma, Meltemi.

- 2013 Returns. Becoming Indigenous in the Twenty-First Century, Cambridge (MA) - London, Harvard University Press.

De Martino E., 1959 L'etnologo e il poeta, in Albino Pierro, Il mio villaggio, Bologna, Cappelli: 147-152.

- 1953 “Etnologia e cultura nazionale negli ultimi dieci anni”, in Società, IX, n. 3: 313-342 (rist. in

E. De Martino, Mondo popolare e magia in Lucania, Roma-Matera, Basilicata editrice 1975: 55-77).

De Rossi A. (a cura di), 2018 Riabitare l'Italia. Le aree interne tra abbandoni e riconquiste, Roma, Donzelli.

Magnaghi A., 2010 Il progetto locale, verso una coscienza di luogo, Torino, Bollati Boringhieri.

- 2017 La conscience du lieu, Paris, Eterotopia France/Rizhome.

- (a cura di), 2012 Il territorio bene comune, Firenze, Firenze University Press.

\section{NOTE FINALI}

1. I. Pisano, "Dare più diritti ai sardi", in l'Unione Sarda, 22 agosto 2018.

2. L'espressione 'porre il centro in periferia' è tratta da alcune note di T.W. Adorno su Walter Benjamin in Note per la letteratura, Torino, Einaudi, 2012, ed è diventata la parola d'ordine della rete dei piccoli paesi, e infine anche una sezione della rivista on line Dialoghi Mediterranei (http:// www.istitutoeuroarabo.it/DM/). Quasi tutte le esperienze di questa rete sono raccontate qui da maggio 2016, a partire da una collaborazione avviata con la pubblicazione del mio testo Casa Lussu. La casa della storia e delle storie, dedicato al lavoro che si fa ad Armungia in Sardegna, per riattivare un paese in crisi demografica drammatica.

3. De Martino peraltro era morto nel 1965 e la generazione del '68 non lo conobbe se non attraverso i libri e gli allievi.

4. In specie Le Sens pratique (1980) che lessi intorno al 1984.

5. Vedi anche il recente De Rossi 2018 e, in particolare, i contributi antropologici di Vito Teti e di Pietro Clemente. Vito Teti è l'antropologo italiano che ha maggiormente riflettuto e scritto sui piccoli paesi e sul tema dell'abbandono e del ritorno, anche con l'uso dei social media.

6. Lares n. 2, 2008, numero speciale su "Gramsci ritrovato", a cura di A. Deias, G.M. Boninelli, E. Testa, ma si veda anche Lares n. 3, 2011, dedicato a Giorgio Baratta: "Prove d'orchestra, Giorgio Baratta e Gramsci tra modernità e contemporaneità", a cura di A. Deias. 


\section{RIASSUNTI}

Negli ultimi decenni, in Italia, di pari passo con l'avanzata della modernizzazione e della globalizzazione, vi è stata la coesistenza di due processi. Il primo è lo spopolamento cui sono andati incontri molti piccoli paesi, in seguito alla massiccia migrazione dei giovani verso i maggiori centri urbani. Il secondo è una generale riscoperta dell'originalità e ricchezza delle culture locali ereditate dal passato, le quali, anche oggi, non solo contraddistinguono il senso dei luoghi e dell'appartenenza ad essi, ma costituiscono una risorsa fondamentale per l'immaginazione del futuro e dell'abitare in esso da parte delle generazioni più giovani. Nella lectio, rivolgendosi idealmente prima di tutto alle generazioni più giovani, l'autore riflette retrospettivamente sul suo contributo, nel corso degli anni, alla discussione di questi temi, soffermandosi in particolare sul suo sforzo di intrecciare la tradizione italiana degli studi di folklore e antropologia con, da una parte, l'attenzione, nei lavori del sociologo Giacomo Becattini e dell'urbanista Alberto Magnaghi, per le nuove forme di "coscienza dei luoghi", e, soprattutto, con il lavoro di James Clifford sui processi del "diventare indigeno nel Ventunesimo Secolo" in Oceania e nelle Americhe.

In the last decades, two big processes have been coexisting in Italy, alongside with the spread of modernization and globalization. The first is the depopulation of many little villages, above all in the inner zone of the country, due to the massive migration of young people to bigger urban centers. The second one is a general rediscovery of the originality and richness of local cultures inherited from the past, that even today mark not only the sense of places and of belonging to them, but constitute a key resource for the younger generations' imagination of the future and of their dwelling in it. In the lecture, first of all ideally addressed to younger generations, the author retrospectively reflects on his contribution, along the work of many years, to these issues, particularly focusing on his attempt to interweave the Italian school of studies of folklore and anthropology with the attention, in the works of sociologist Giacomo Becattini and the urbanist Alberto Magnaghi, for the new forms of "consciousness of places", and above all, with James Clifford's work on the processes of "becoming indigenous in the XXI ${ }^{\text {th }}$ Century" in Oceania and the Americas.

\section{INDICE}

Keywords : sense of place, center/peripheries, imagination of future, cultural homeland, new indigenity

Parole chiave : senso del luogo, centro/periferie, immaginazione del futuro, patria culturale, nuova indigenità

\section{AUTORE}

\section{PIETRO CLEMENTE}

Presidente onorario della Società Italiana per la Museografia e i Beni DemoEtnoAntropologici (SIMBDEA) 